\title{
La violencia en las mujeres usuarias de los servicios de salud en el IMSS y la SSA
}

\author{
Héctor Gómez-Dantés, MC, (I) José Luis Vázquez-Martínez, MC, ${ }^{(1)}$ \\ Sonia B. Fernández-Cantón, Dra. ${ }^{(1)}$
}

\author{
Gómez-Dantés H,Vázquez-Martínez JL, \\ Fernández-Cantón SB. \\ La violencia en las mujeres usuarias de los servicios \\ de salud en el IMSS y la SSA. \\ Salud Publica Mex 2006;48 supl 2:S279-S287.
}

\section{Resumen}

Objetivo. Comparar la prevalencia y determinar los factores de riesgo asociados a la violencia entre las mujeres derechohabientes del IMSS y sin servicios de seguridad social (SSS). Material y métodos. Se analizó la información sobre violencia doméstica de la ENVIM notificada por las mujeres usuarias de los servicios de salud del IMSS y de la población femenina sin servicios de seguridad social SSA y seguro popular. El análisis bivariado y multivariado se realizó en STATA V.7. Resultados. La prevalencia en mujeres derechohabientes del IMSS de violencia psicológica fue 18\%; seguida por la física (9.1\%); la sexual (6.7\%) y la económica (5\%). En mujeres sin seguridad social fue:psicológica (21.4\%); física (I0.5\%); sexual (7.5\%) y económica (5.2\%). Las mujeres entre 25 y 44 años, con nivel secundaria o menor instrucción educativa, casadas, sufren de mayor violencia doméstica en ambas poblaciones. Los factores de riesgo identificados para los cuatro tipos de violencia fueron el consumo de alcohol en la pareja, las edades jóvenes, el estar casadas o en unión libre y el antecedente de violencia ejercida por los padres u otros miembros de la familia. Conclusiones. La violencia es ligeramente mayor en mujeres sin seguridad social. El consumo diario de alcohol por su pareja es un factor de riesgo muy importante para cualquier tipo de violencia, en particular cuando el consumo es diario o casi habitual. La detección de la violencia doméstica en los servicios de salud es indispensable para conocer su magnitud como problema social.

Palabras clave: violencia doméstica; género; alcohol; embarazo; México
Gómez-Dantés H,Vázquez-Martínez JL, Fernández-Cantón SB.

Violence in Mexican women using

public health services.

Salud Publica Mex 2006;48 suppl 2:S279-S287.

\section{Abstract}

Objective. To compare the prevalence of violence and determine its risk factors among women who use Mexican Social Services (IMSS) clinics and do not have access to social security services. Material and Methods. Sociodemographic data linked to domestic violence reported by women attending the Mexican Institute of Social Security (IMSS) health services was analyzed. Bivariate and multivariate analysis was performed using STATA V.7. Results. Psychological violence in IMSS women was $18 \%$ followed by physical violence $(9.1 \%)$, sexual $(6.7 \%)$ and economic $(5 \%)$. Prevalence of violence in women with no social security care was psychological (2I.4\%), physical (I0.5\%), sexual (7.5\%) and economical (5\%). Women between 25 and 44 years of age with basic schooling and married and with family background of violence were the most affected. The daily consumption of alcohol by their partners was an important predictor of domestic violence. Conclusion. Violence in women with no social security is higher. Partner's alcohol intake pattern is an important risk factor. Detection of domestic violence in the clinical setting is necessary to recognize its real magnitude as a social problem.

Keywords: domestic violence; gender; alcohol; pregnancy; Mexico

(I) División Técnica de Información Estadística en Salud (DTIES), Dirección de Prestaciones Médicas, IMSS, México.

Fecha de recibido: 18 de enero de 2005 - Fecha de aprobado: 15 de noviembre de 2005

Solicitud de sobretiros: Héctor Gómez Dantés, División Técnica de Información Estadística en Salud, Dirección de Prestaciones Médicas IMSS.

Durango 289, 6o. piso, colonia Roma, 06700 México, D.F.

Correo electrónico: hector.gomez@imss.gob.mx 
D esde el campo de la salud pública, el estudio de la violencia contra la mujer impone desafíos diversos para su identificación, atención y prevención. La violencia tiene diferentes rostros que se expresan bajo un amplio espectro de daños, siendo los más evidentes las lesiones físicas que van del maltrato hasta la muerte. Sin embargo, el daño psicológico y las repercusiones corporales que encubren a la violencia pueden causar lesiones, enfermedades diversas y discapacidades permanentes, lo que la convierten en una de las principales determinantes de la salud de las mujeres. Las lesiones intencionales y no intencionales figuran dentro de las principales causas de muerte y sus consecuencias se enmascaran bajo múltiples diagnósticos en la demanda de consulta de urgencias y medicina familiar. Diferentes estudios informan que las mujeres son las víctimas mas frecuentes de la violencia doméstica y que sus parejas (marido, novio, compañero) son los responsables de la agresión. ${ }^{1-5}$ Las consecuencias de la violencia en las mujeres se manifiestan como traumatismos de diversa severidad, infecciones de transmisión sexual, incluyendo la infección por VIH, embarazos no deseados, abortos, partos prematuros, hijos con bajo peso, depresión, suicidio, alcoholismo, etc. ${ }^{6,7}$ Este espectro provoca que la violencia en las mujeres y su diagnóstico estén subregistrados, a pesar de tener bien documentada la mayor demanda de servicios de salud y de consulta en los servicios de urgencias por parte de las mujeres que sufren de violencia familiar. ${ }^{8}$

En 2002, la violencia interpersonal fue en el mundo la sexta causa principal de defunción entre las personas de 15 a 44 años. Aproximadamente 40 millones de niños son objetos de maltrato cada año mientras que las violaciones sexuales y la violencia doméstica son responsables de 5 a $16 \%$ de los años de vida sana perdidos en las mujeres en edad fértil. ${ }^{9}$ Las estimaciones sobre la magnitud de la violencia varían según la metodología que se utiliza para el estudio y la medición de la misma. En el Reino Unido se notifican tasas de violencia doméstica alguna vez en la vida de 12 a $46 \%,{ }^{10}$ mientras que en Estados Unidos de América la prevalencia notificada es de $36.9 \%$ y se estima que en ese país alrededor de 3 mil 300 mujeres son golpeadas severamente por sus esposos. ${ }^{11,12} \mathrm{En}$ muchas partes del mundo los accidentes y los homicidios figuran entre las principales causas de muerte en los diferentes grupos de edad. En el caso concreto de las mujeres, la mayoría de las muertes por homicidio ocurren en el hogar y su pareja es la persona más frecuentemente involucrada en ese desenlace violento. ${ }^{13,14}$ Por otro lado, el abuso y las violaciones sexuales, el maltrato físico y psicológico, cierta proporción de las infecciones de transmisión sexual, de los embarazos no deseados, del aborto, el bajo peso al nacer, ${ }^{15}$ la drogadicción, el alcoholismo, la depresión, ${ }^{16}$ el suicidio, el homicidio y las lesiones son expresiones clínicas de la violencia hacia la mujer. Tan diversas manifestaciones hacen muy complicada la identificación de la violencia en el escenario clínico.

La Encuesta Nacional sobre Violencia contra las Mujeres 2003 (ENVIM, 2003) levantada por la Secretaría de Salud (SSA) constituye un esfuerzo singular por cuantificar la magnitud de la violencia hacia las mujeres en el país. Esto significa que conocer el panorama de la violencia en México cobra hoy la importancia de un problema de salud pública prioritario y que las instituciones públicas y privadas encargadas de la salud de la población deben estar atentas a este problema. El objetivo del presente trabajo es describir la prevalencia, así como identificar algunos factores asociados a los diferentes tipos de violencia en las mujeres usuarias de los servicios de salud en el Instituto Mexicano del Seguro Social (IMSS) en comparación con mujeres que carecen de servicios de seguridad social.

\section{Material y métodos}

Los resultados descritos provienen del análisis secundario de la ENVIM 2003, realizada a las mujeres de 15 años y más, usuarias de los servicios de salud en las unidades de primero y segundo nivel de atención del IMSS, así como las de los servicios de la SSA, lo que incluye a mujeres con seguro popular. Las mujeres seleccionadas fueron aquellas que demandaron atención de las unidades médicas seleccionadas y aceptaron participar en el estudio después de firmar una carta de consentimiento informado.

La violencia contra la mujer fue basada en la definición planteada por la Asamblea General de las Naciones Unidas sobre la Eliminación de la Violencia en contra de la Mujer. La operacionalización se realizó en cuatro tipos de violencia y cada uno de estos se consideró como variable dependiente en los modelos bivariados y multivariados. La definición de violencia física incluye a la mujer a quien alguna vez su novio, esposo, compañero o última pareja la haya empujado a propósito, jaloneado, torcido el brazo, le haya pegado con el puño o la mano, pateado, golpeado con algún palo, cinturón u otro objeto doméstico. La violencia psicológica sucede cuando el hombre la haya rebajado o menospreciado a solas o frente a otra personas, insultado, celado, le haya dicho que es poco atractiva o fea, que haya golpeado la pared o mueble. La violencia sexual se refiere a cuando el hombre le haya exigido tener relaciones sexuales, o si la amenazó con irse 
con otras mujeres si no accedía a tener relaciones sexuales con él, o haya usado la fuerza física para tenerlas. La violencia económica se describe cuando el hombre la haya controlado con no darle o quitarle el dinero, haya hecho uso de pertenencias en contra de su voluntad.

De las 17 secciones que consta el cuestionario, se tomaron las características demográficas de la encuestada (edad, escolaridad, estado civil, estrato socioeconómico), la identificación de la derechohabiencia de servicios de salud, la salud general y personal, el estado emocional, el consumo de alcohol en su pareja y el antecedente de violencia familiar. La entrevista se realizó dentro de las unidades de salud, en un consultorio adecuado para contar con privacidad, comodidad y durante el periodo comprendido del 1 de noviembre del 2002 al 25 de marzo del 2003.

El tamaño de la muestra se calculó para contar con representatividad estatal y nacional y con una estratificación hecha con base en la distribución proporcional de consultorios médicos en las unidades de las instituciones de salud, al interior de cada entidad federativa, según los datos de la ENSA 2000. El número de unidades seleccionadas por entidad fue de 10 y la muestra de 820 mujeres usuarias se tomó de manera sistemática y se distribuyó acorde a la presencia institucional del IMSS, del Instituto de Seguridad y Servicios Sociales de los Trabajadores del Estado (ISSSTE) y la SSA. Las instituciones consideradas en este estudio fueron el IMSS y la SSA con representatividad nacional. Los detalles sobre el levantamiento de la encuesta, la selección de las mujeres usuarias, la capacitación del personal y el diseño de los instrumentos están claramente descritos en el informe de resultados preliminares de la ENVIM. ${ }^{17}$

La derechohabiencia se obtuvo de la ENVIM en la sección de características demográficas y del cuestionario sobre utilización de servicios de salud. La frecuencia de las variables dependientes e independientes de las mujeres derechohabientes del IMSS se comparó con la de la población encuestada sin derechohabiencia (SSA y con seguro popular) a través de porcentajes. Como prueba estadística se utilizó la prueba de ji cuadrada (cuadros I y II). En el cuadro III se describe la frecuencia de cada tipo de violencia (porcentajes), considerando cada una de las variables independientes y por tipo de población (IMSS y sin seguridad social). El análisis multivariado se realizó a través de la técnica estadística de regresión logística, en donde la variable dependiente se construyó con cada uno de los tipos de violencia y las variables independientes fueron: edad (15 a 24, 25 a 44, 45 a 64, y 65 y más años); estado civil (soltera, casada, divorciada, separada, viuda y unión libre); escolaridad (sin instrucción y preescolar, primaria completa, educación media y superior); estrato socioeconómico (muy bajo, bajo, medio y alto); consumo de alcohol por la pareja (diario, 1 a 2 veces por semana, 1 a 3 veces al mes, nunca); problema de salud actual (sí/no); malestar emocional (alterado y no alterado) y el antecedente de violencia por parte de algún familiar (padre o madre, otros y nadie). El análisis de la información se realizó con el paquete de STATA V7.* Para el modelo multivariado las variables de estado civil y estrato se compactaron para asegurar una probable relación con la variable dependiente. La incorporación de variables se hizo de un modelo saturado a un modelo parsimonioso.

\section{Resultados}

De las 26042 de mujeres usuarias de servicios de salud en todo el país, que la ENVIM logró entrevistar, se seleccionó a 10787 mujeres derechohabientes del IMSS (41.4\%) y a 12423 mujeres sin seguridad social (47.7), abarcando un universo de estudio de 23210 mujeres (89\% del universo de la encuesta). La proporción de mujeres en ambas poblaciones fue mayor en el grupo de edad de 25 a 44 años, en las derechohabientes del IMSS, fue de $52 \%$ y para las que no tienen seguridad social, de $51.1 \%$. En general, no se observan diferencias marcadas entre la estructura poblacional de ambos grupos. En cuanto a las condiciones sociodemográficas de las encuestadas, las mujeres usuarias del IMSS cuentan con mejores niveles de escolaridad a partir del nivel intermedio y superior; la frecuencia de mujeres sin instrucción fue casi dos veces mayor en las mujeres sin seguridad social (11.9 vs 6.5\%). En cuanto al estado civil se puede observar que $62 \%$ de las usuarias del IMSS están casadas y sólo $11.9 \%$ en unión libre, a diferencia de 49.2 y $25 \%$, respectivamente. La proporción de solteras, divorciadas, separadas y viudas es muy similar en ambos grupos. El estrato socioeconómico de las usuarias derechohabientes del IMSS es mayor que el de las mujeres sin seguridad social. El informe de problemas de salud fue mayor en las mujeres usuarias del IMSS (55\%) que en mujeres sin seguridad social $(46.7 \%)$, aunque la percepción del estado de salud, el estado emocional y el antecedente de violencia familiar es muy similar en los dos grupos. En cuanto al consumo de alcohol por la pareja de la mujer, fue similar en ambos grupos, con una mayor frecuencia para un consumo de 1 a 3 veces por mes $u$ ocasionalmente.

* Stata 7.0 Software Stata Corp, Collage Station. TX. 


\section{Cuadro I \\ CONDICIONES SOCIODEMOGRÁFICAS Y DE SALUD DE LAS MUJERES USUARIAS SEgún deRECHOHABIENCIA. ENVIM 2003, MéXICO}

\begin{tabular}{|c|c|c|c|c|c|c|c|}
\hline & & \multicolumn{2}{|c|}{ IMSS } & \multicolumn{2}{|c|}{ Sin seguridad social } & \multicolumn{2}{|c|}{ Total } \\
\hline & & $n$ & $\%$ & $n$ & $\%$ & $n$ & $\%$ \\
\hline Total de usuarias & & 10787 & 100.0 & 12423 & 100.0 & 23210 & 100.0 \\
\hline \multirow[t]{6}{*}{ Grupo de edad (años) } & $15-24$ & 2349 & 21.8 & 3441 & 27.7 & 5790 & 24.9 \\
\hline & $25-44$ & 5664 & 52.5 & 6345 & 51.1 & 12009 & 51.7 \\
\hline & $45-64$ & 2325 & 21.6 & 2222 & 17.9 & 4547 & 19.6 \\
\hline & 65 y más & 446 & 4.1 & 412 & 3.3 & 858 & 3.7 \\
\hline & No especificado & 3 & 0.0 & 3 & 0.0 & 6 & 0.0 \\
\hline & & $\chi^{2}=133.57$ & $p=0.0001$ & & & & \\
\hline \multirow[t]{6}{*}{ Grado de escolaridad } & Sin instrucción y preescolar & 705 & 6.5 & | 477 & 11.9 & 2182 & 9.4 \\
\hline & Primaria completa & 3793 & 35.2 & 5734 & 46.2 & 9527 & 41.0 \\
\hline & Secundaria y carrera técnica & 4328 & 40.1 & 3991 & 32.1 & 8319 & 35.8 \\
\hline & Preparatoria y superior & 1938 & 18.0 & 1180 & 9.5 & 3118 & 13.4 \\
\hline & No especificado & 23 & 0.2 & 41 & 0.3 & 64 & 0.3 \\
\hline & & $\chi^{2}=757.11$ & $p=0.0001$ & & & & \\
\hline \multirow[t]{8}{*}{ Estado civil } & Casada & 6716 & 62.3 & 6114 & 49.2 & 12830 & 55.3 \\
\hline & Unión libre & 1279 & 11.9 & 3108 & 25.0 & 4387 & 18.9 \\
\hline & Soltera & 1130 & 10.5 & 1249 & 10.1 & 2379 & 10.2 \\
\hline & Vivió en unión libre & 138 & 1.3 & 207 & 1.7 & 345 & 1.5 \\
\hline & Separada & 672 & 6.2 & 934 & 7.5 & 1606 & 6.9 \\
\hline & Viuda & 650 & 6.0 & 652 & 5.2 & 1302 & 5.6 \\
\hline & Divorciada & 202 & 1.9 & 159 & 1.3 & 361 & 1.6 \\
\hline & & $\chi^{2}=746.79$ & $p=0.0001$ & & & & \\
\hline \multirow[t]{6}{*}{ Estrato socioeconómico } & Muy bajo & 3211 & 29.8 & 7915 & 63.7 & II I26 & 47.9 \\
\hline & Bajo & 5420 & 50.2 & 3712 & 29.9 & 9132 & 39.3 \\
\hline & Medio & 1586 & 14.7 & 643 & 5.2 & 2229 & 9.6 \\
\hline & Alto & 535 & 5.0 & 119 & 1.0 & 654 & 2.8 \\
\hline & No especificado & 35 & 0.3 & 34 & 0.3 & 69 & 0.3 \\
\hline & & 7 & & & & & \\
\hline \multirow[t]{3}{*}{ Problemas de salud } & Sí & 5916 & 54.8 & 5801 & 46.7 & 11717 & 50.5 \\
\hline & No & 4871 & 45.2 & 6622 & 53.3 & 11493 & 49.5 \\
\hline & & $\chi^{2}=153.34$ & $p=0.0001$ & & & & \\
\hline Percepción del & Muy buena & 426 & 3.9 & 471 & 3.8 & 897 & 3.9 \\
\hline \multirow[t]{5}{*}{ estado de salud } & Buena & 4659 & 43.2 & 5338 & 43.0 & 9997 & 43.1 \\
\hline & Regular & 4539 & 42.1 & 5346 & 43.0 & 9885 & 42.6 \\
\hline & Mala & 990 & 9.2 & $111 \mathrm{I}$ & 8.9 & 2101 & 9.1 \\
\hline & Muy mala & 173 & 1.6 & 157 & 1.3 & 330 & 1.4 \\
\hline & & $\chi^{2}=6.72$ & $p=0.15 \mid$ & & & & \\
\hline \multirow[t]{3}{*}{ Estado emocional } & Alterado & 1678 & 15.6 & 1905 & 15.3 & 3583 & 15.4 \\
\hline & No alterado & 9109 & 84.4 & 10518 & 84.7 & 19627 & 84.6 \\
\hline & & $\chi^{2}=0.0619$ & $p=0.970$ & & & & \\
\hline Antecedente de & Nadie & 9272 & 86.0 & 10670 & 85.9 & 19942 & 85.9 \\
\hline \multirow[t]{3}{*}{ violencia familiar } & Padre o madre & 778 & 7.2 & 894 & 7.2 & 1672 & 7.2 \\
\hline & Otros & 737 & 6.8 & 859 & 6.9 & 1596 & 6.9 \\
\hline & & $\chi^{2}=0.2166$ & $p=0.642$ & & & & \\
\hline Consumo de alcohol & Todos o casi todos los días & 607 & 5.6 & 771 & 6.2 & I 378 & 5.9 \\
\hline \multirow{4}{*}{ en la pareja } & Una o dos veces por semana & $|36|$ & 12.6 & 1510 & 12.2 & 2871 & 12.4 \\
\hline & Una a tres veces al mes & 5785 & 53.6 & 6646 & 53.5 & 12431 & 53.6 \\
\hline & Nunca & 1463 & 13.6 & 1705 & 13.7 & 3168 & 13.6 \\
\hline & No especificado & $|57|$ & 14.6 & | 79| & 14.4 & 3362 & 14.5 \\
\hline
\end{tabular}

Fuente: Encuesta Nacional sobre Violencia contra las Mujeres 2003 (ENVIM) 


\section{Cuadro II \\ Prevalencia de violencia en mujeres usuarias DE SERVICIOS DE SALUD, POR TIPO Y DERECHOHABIENCIA}

\begin{tabular}{|c|c|c|c|c|c|c|c|}
\hline & & & & $\begin{array}{r}\text { Sin seg } \\
\text { soc }\end{array}$ & $\begin{array}{l}\text { uridad } \\
\text { ial }\end{array}$ & & \\
\hline & & $n$ & $\%$ & $n$ & $\%$ & $n$ & $\%$ \\
\hline Psicológica & Sí & 1865 & 18.0 & 2551 & 21.4 & 4416 & 19.8 \\
\hline & No & 8470 & 82.0 & 9385 & 78.6 & 17855 & 80.2 \\
\hline & Total & 10335 & 100.0 & 11936 & 100.0 & $2227 \mid$ & 100.0 \\
\hline
\end{tabular}

\begin{tabular}{|c|c|c|c|c|c|c|}
\hline Física & 942 & 9.1 & 1250 & 10.5 & 2192 & 9.8 \\
\hline No & 9391 & 90.9 & 10685 & 89.5 & 20076 & 90.2 \\
\hline Total & 10333 & 100.0 & 11935 & 100.0 & 22268 & 100.0 \\
\hline
\end{tabular}

\begin{tabular}{rlrrrrrr} 
Sexual & Si & 693 & 6.7 & 901 & 7.5 & 1594 & 7.2 \\
\hline & No & 9639 & 93.3 & 11034 & 92.5 & 20673 & 92.8 \\
\hline Total & 10332 & 100.0 & 11935 & 100.0 & 22267 & 100.0 \\
\hline$\chi^{2}=5.9062$ & $p=0.015$ & & & &
\end{tabular}

\begin{tabular}{rlrrrrrr} 
Económica & Si & 521 & 5.0 & 622 & 5.2 & 1143 & 5.1 \\
\hline & No & 9810 & 95.0 & $1 \mid 1313$ & 94.8 & 21123 & 94.9 \\
\hline Total & 10331 & 100.0 & $1 \mid 935$ & 100.0 & 22266 & 100.0 \\
\hline$\chi^{2}=0.3228$ & $p=0.570$ & & & &
\end{tabular}

Fuente: Encuesta Nacional sobreViolencia contra las Mujeres 2003 (ENVIM)

La magnitud de la violencia en la población derechohabiente del IMSS presenta diferentes matices. Por tipos de violencia, la psicológica fue la más frecuente $(18 \%)$, seguida por la física $(9.1 \%)$, la sexual $(6.7 \%)$ y la económica (5\%). La prevalencia de violencia fue discretamente mayor en las mujeres sin seguridad social: la psicológica fue la más frecuente $(21.4 \%)$, seguida por la violencia física $(10.5 \%)$, sexual $(7.5 \%)$ y económica $(5.2 \%)$ (cuadro II).

Los tipos de violencia en las usuarias de los servicios de salud muestran diferencias interesantes desde la perspectiva de la derechohabiencia (cuadro III). En cuanto a la edad, $60 \%$ de las mujeres afectadas para todos los tipos de violencia y para ambos grupos se encuentran entre los 25 y los 44 años. No obstante, la violencia en las adolescentes y mayores de 60 años se presenta en magnitudes que merecen especial atención. Por ejemplo, 20\% de los casos de violencia ocurren en las mujeres mas jóvenes (15 a 24 años) derechohabientes del IMSS y en $26.5 \%$ de las mujeres sin seguridad social . La violencia física se detectó en $21 \%$ de las derechohabientes y casi en 30\% de las que carecen de seguridad social, mientras que la sexual se presentó en
16 y $24.4 \%$, respectivamente. La violencia económica se presentó en magnitudes similares en ambos grupos (17.1 vs. $20.4 \%$ ).

Un evento que destaca es que la distribución de la violencia en las mujeres derechohabientes predomina en aquellas con educación media y superior, mientras que en las mujeres sin seguridad social la violencia es mayor en aquellas con educación básica. Este patrón se presenta en todos los tipos de violencia. En cuanto al estado civil, se encontró que en las mujeres derechohabientes casadas se concentra una mayor proporción de casos de violencia, y que en las mujeres sin seguridad social predomina en las que establecen su relación en unión libre. Por estrato socioeconómico, los estratos más bajos para las mujeres sin seguridad social concentran $65 \%$ de los casos de violencia, mientras que en las mujeres derechohabientes los casos se concentran en los estratos bajo y medio. El informe de problemas de salud en las mujeres con violencia es particularmente alto: se ubica entre 30 y $60 \%$ en las mujeres derechohabientes y $50 \%$ en las mujeres sin seguridad social, y contrasta con la percepción del estado de salud, pues entre 30 y 40\% de las mujeres indicaron estar en buenas o muy buenas condiciones de salud. Con relación a estado emocional, aproximadamente $30 \%$ presentaban alteraciones, con magnitudes similares para los cuatro tipos de violencia y frecuencias similares entre ambos grupos. Entre 25 y 30\% de las mujeres con algún tipo de violencia tenían antecedente de violencia familiar, ejercida por los padres $\mathrm{u}$ otros miembros de la familia. En cuanto al consumo de alcohol, entre 80 y $90 \%$ de las mujeres que sufrieron algún tipo de violencia manifestaron consumo de alcohol por parte de su pareja, con frecuencias similares entre ambos grupos de mujeres (cuadro III). Estas diferencias también están marcadas por la diferente distribución de las mujeres usuarias de cada institución, al interior de cada categoría.

El análisis descriptivo pone de relieve la participación e influencia de múltiples factores en el ejercicio de la violencia y la intrincada relación entre ellos puede favorecerla u obstaculizarla. A través del análisis multivariado se combinaron las relaciones entre los factores involucrados y se controló el efecto que tienen sobre la acción de los otros. En los modelos para cada tipo de violencia se incorporaron el consumo de alcohol por la pareja, la escolaridad, la edad, el estado civil, la existencia de un problema de salud reciente, el estrato socioeconómico, el antecedente de violencia familiar y la derechohabiencia (cuadro IV). Sin lugar a dudas, la asociación del alcohol con la violencia es muy importante y el riesgo de sufrir cualquier tipo de violencia se incrementa sustancialmente cuando el consumo de 
Cuadro III

Características sociodemográficas de las mujeres QUe SUfren Violencia, SEgún EL TIPO DE LA MISMA Y DERECHOHABIENCIA. ENVIM, 2003, MÉXICO

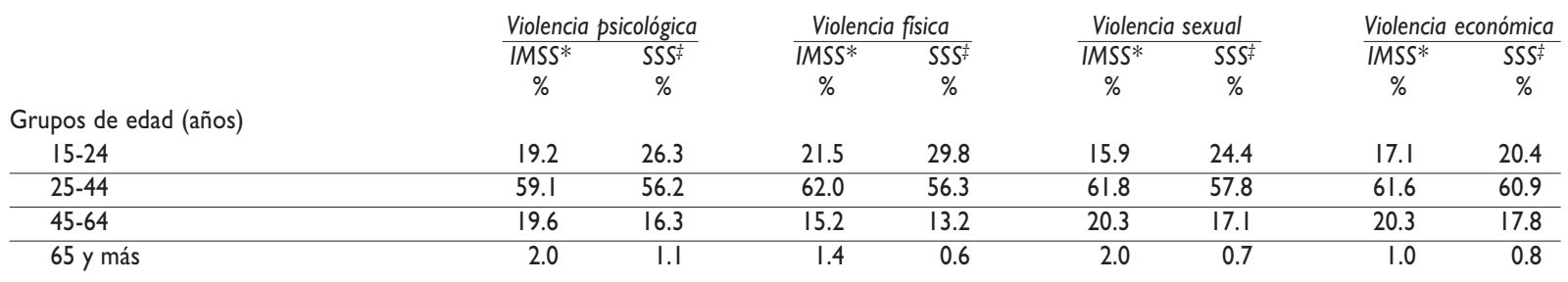

Grado de escolaridad

\begin{tabular}{lrrrrrrrr} 
Sin instrucción y preescolar & 8.2 & 12.3 & 7.7 & 11.1 & 7.8 & 11.4 & 7.9 & 12.3 \\
\hline Primaria completa & 37.9 & 49.3 & 38.2 & 47.0 & 42.5 & 53.5 & 42.0 & 48.6 \\
\hline Secundaria y carrera técnica & 40.6 & 30.5 & 42.2 & 33.5 & 38.8 & 27.1 & 38.8 & 31.0 \\
\hline Preparatoria y superior & 13.3 & 7.9 & 11.9 & 8.5 & 10.9 & 8.0 & 11.3 & 8.1
\end{tabular}

\begin{tabular}{lrrrrrrrr}
$\begin{array}{l}\text { Estado civil } \\
\text { Casada }\end{array}$ & 69.9 & 54.4 & 65.8 & 49.9 & 67.7 & 50.3 & 65.6 & 48.4 \\
\hline Unión libre & 15.0 & 31.5 & 15.6 & 33.3 & 14.7 & 31.2 & 14.4 & 33.1 \\
\hline Soltera & 3.6 & 3.5 & 3.3 & 3.0 & 2.7 & 3.1 & 1.9 & 1.8 \\
\hline Vivió en unión libre & 0.9 & 1.5 & 1.1 & 1.5 & 1.2 & 2.1 & 1.3 & 1.6 \\
\hline Separada & 7.8 & 7.4 & 10.8 & 10.2 & 10.7 & 11.1 & 12.7 & 12.7 \\
\hline Viuda & 1.2 & 1.0 & 1.3 & 1.3 & 1.3 & 1.1 & 1.5 & 1.4 \\
\hline Divorciada & 1.6 & 0.7 & 2.1 & 0.8 & 1.7 & 1.1 & 2.5 & 1.0
\end{tabular}

Estrato socioeconómico

\begin{tabular}{lrrrrrrrr} 
Muy bajo & 33.1 & 65.8 & 33.6 & 65.4 & 36.2 & 66.5 & 35.5 & 65.1 \\
\hline Bajo & 52.4 & 30.1 & 53.3 & 30.3 & 51.2 & 29.7 & 52.0 & 30.4 \\
\hline Medio & 11.6 & 3.4 & 10.8 & 3.8 & 10.1 & 3.0 & 9.2 & 4.0 \\
\hline Alto & 2.8 & 0.6 & 2.2 & 0.4 & 2.4 & 0.8 & 3.3 & 0.5
\end{tabular}

Problemas de salud

$\begin{array}{lllllllll}\text { Sí } & 61.3 & 51.9 & 37.8 & 53.3 & 33.6 & 56.8 & 66.4 & 57.4\end{array}$

Percepción del estado de salud

\begin{tabular}{lrrrrrrrr} 
Muy buena & 3.9 & 4.5 & 3.1 & 2.7 & 2.9 & 2.1 & 4.4 & 1.3 \\
\hline Buena & 35.3 & 35.8 & 34.5 & 36.9 & 30.0 & 32.1 & 29.2 & 34.9 \\
\hline Regular & 47.3 & 47.1 & 48.5 & 46.2 & 49.2 & 48.2 & 50.3 & 44.9 \\
\hline Mala & 11.5 & 11.3 & 11.9 & 12.2 & 14.3 & 15.0 & 13.6 & 16.4 \\
\hline Muy mala & 1.9 & 1.4 & 2.0 & 1.9 & 3.6 & 2.7 & 2.5 & 2.6
\end{tabular}

Estado emocional

\begin{tabular}{lllllllll} 
Alterado & 30.2 & 26.7 & 35.9 & 31.6 & 37.8 & 34.6 & 39.5 & 38.1 \\
\hline No alterado & 69.8 & 73.3 & 64.1 & 68.4 & 62.2 & 65.4 & 60.5 & 61.9
\end{tabular}

Antecedente de violencia familiar

\begin{tabular}{lcccccccc} 
Nadie & 75.2 & 77.3 & 73.6 & 74.2 & 71.7 & 74.9 & 69.1 & 69.3 \\
\hline Padre o madre & 11.8 & 10.7 & 12.4 & 11.4 & 12.0 & 9.4 & 15.4 & 13.8 \\
\hline Otros & 13.0 & 12.0 & 14.0 & 14.3 & 16.3 & 15.6 & 15.5 & 16.9
\end{tabular}

Consumo de alcohol en la pareja

\begin{tabular}{lrrrrrrrr} 
Todos o casi todos los días & 12.7 & 10.1 & 14.8 & 13.4 & 17.7 & 17.0 & 18.2 & 19.1 \\
\hline Una o dos veces por semana & 21.5 & 20.5 & 22.9 & 24.1 & 24.4 & 24.8 & 25.0 & 24.6 \\
\hline Una a tres veces al mes-ocasionalmente & 50.6 & 55.3 & 47.8 & 49.5 & 45.2 & 45.0 & 44.1 & 45.3 \\
\hline Nunca & 9.3 & 7.9 & 8.6 & 6.8 & 7.5 & 6.8 & 7.9 & 6.1 \\
\hline No especificado & 6.0 & 6.2 & 5.9 & 6.2 & 5.2 & 6.5 & 4.8 & 4.8
\end{tabular}

* IMSS, Instituto Mexicano del Seguro Social

‡ SSS, sin seguridad social

Fuente: Encuesta Nacional sobre Violencia contra las Mujeres 2003 (ENVIM) 


\section{Cuadro IV}

\section{Riesgos de PADECER DIFERENTES TIPOS DE VIOLENCIA DE LAS MUJERES USUARIAS DE Los SERVICIOS DE SALUd. ENVIM, 2003, MéXico}

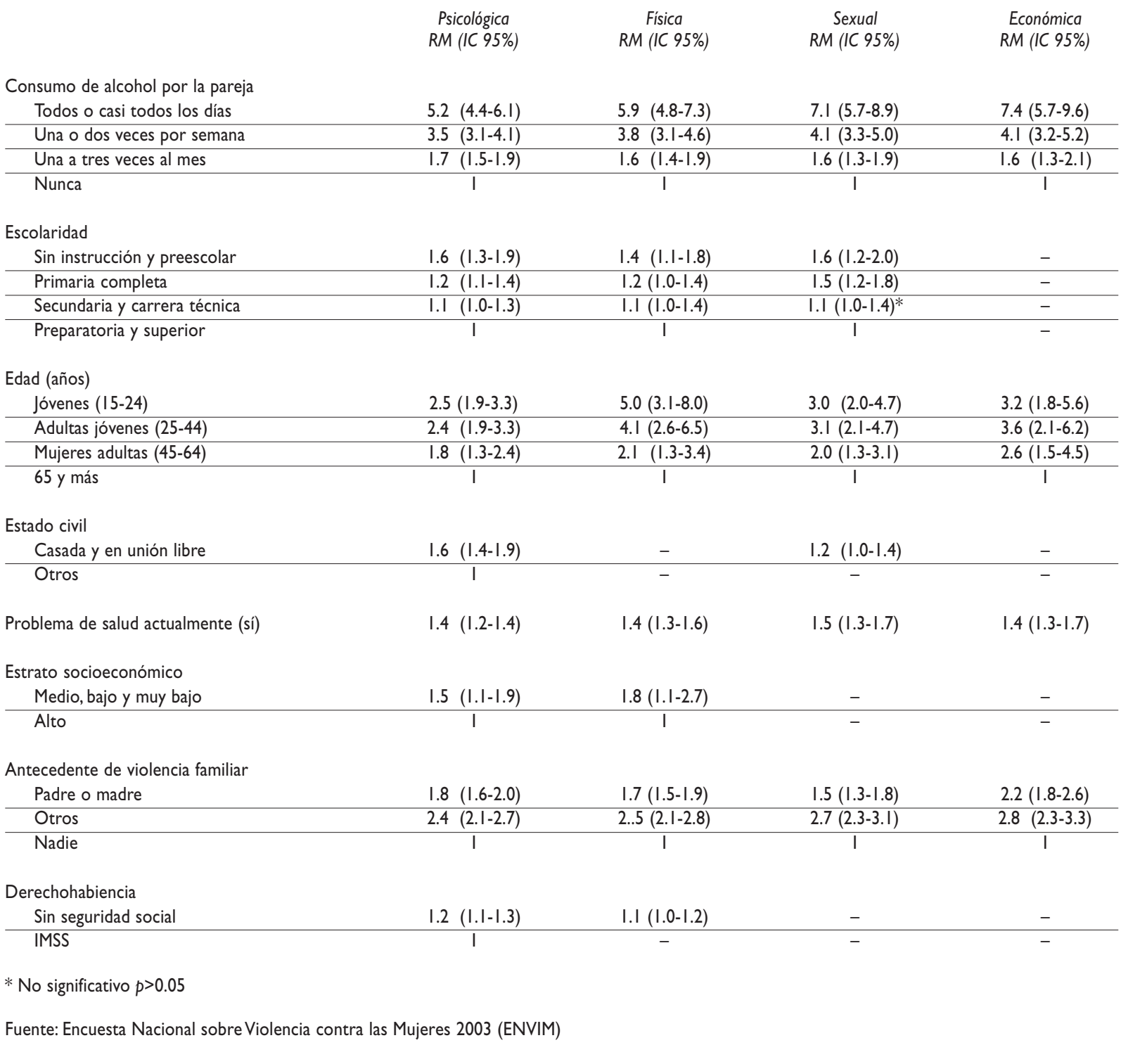

alcohol por parte de la pareja ocurre todos los días o de una a dos ocasiones por semana. El efecto de la escolaridad sobre la violencia también es muy importante, ya que a mayor educación menor riesgo de sufrir violencia, mientras que el analfabetismo en la mujer prácticamente duplica el riesgo de sufrir violencia psicológica, física y sexual. La violencia en los cuatro tipos estudiados decrece con la edad, aunque existe en la adolescencia y perdura hasta las mujeres adultas de
45 a 64 años. En cuanto al estado civil, el riesgo de violencia psicológica fue mayor en las mujeres casadas y en unión libre, que son las que tiene pareja de manera permanente. Las mujeres con mejor nivel socioeconómico sufren de menos violencia que sus contrapartes. La existencia de un problema de salud en el momento de la entrevista está discretamente asociada a la incidencia de violencia, ya que fue más frecuente en las mujeres que notificaron problemas de salud que en las 
que se dijeron estar sanas. Por último, el antecedente de violencia familiar influyó en la prevalencia de violencia, sobre todo cuando la ejercían otros familiares diferentes al padre o la madre (padrastro o madrastra y otros). La carencia de seguridad social coloca a la mujer en un nivel mayor de riesgo, aunque no llega a ser tan importante como el consumo de alcohol, la escolaridad y el estado civil.

\section{Discusión}

Aunque la prevalencia de la violencia dentro de la población derechohabiente del IMSS fue menor a la notificada por las mujeres usuarias de otros servicios de salud, ambas poblaciones comparten muchas de las determinantes de la violencia. Más de la mitad de las mujeres encuestadas informaron acerca del antecedente de algún evento violento en su vida y el rango de dicha prevalencia en diversos estudios realizados en México va de 20 a 57\%. ${ }^{2}$ La violencia psicológica fue la más frecuente (19.8\%) seguida por la física $(9.8 \%)$ y la sexual $(7.2 \%)$. Este patrón es muy similar a lo informado por otros estudios en México, aunque varían las magnitudes dependiendo del momento, el lugar y la población encuestada. Por ejemplo, las prevalencias en las mujeres entrevistadas en un hospital pediátrico del IMSS fueron 98,50 y 16\%, ${ }^{4}$ mientras que en otro estudio realizado en la ciudad de Durango se registraron prevalencias de 39,40 y $42 \%,{ }^{18}$ respectivamente. Estas cifras contrastan con lo notificado en la Ciudad de México ( 6,9 y $1.4 \%)^{3}$ o lo informado en áreas urbanas por la encuesta del INEGI $(99,11$ y $1 \%) .{ }^{19}$ Las diferencias en los resultados hablan de las peculiaridades de la población encuestada y los instrumentos utilizados para identificar el tipo de violencia; sin embargo, todos los estudios alcanzan a resaltar la magnitud del problema. La violencia contra las mujeres es un problema de salud tanto o más importante que muchos otros para los cuales se cuentan con recursos y estrategias de detección, control y prevención bien establecidas.

En el modelo multivariado el efecto de la edad se modifica al interactuar con el resto de las variables e inclusive aparece como factor de riesgo a edades más jóvenes comparadas con las mujeres adultas mayores. ${ }^{1,6}$ Esto magnifica la relevancia del problema pues nos puede indicar que las generaciones jóvenes responden con mayor violencia a la frustración o a la falta de oportunidades y que su prevalencia tiende a aumentar en la medida que se engrosa la estructura de la población y crece el índice de dependencia. También es muy probable que las mujeres adultas y las menos educadas no respondan y tampoco compartan con tanta facili- dad sus experiencias en las indagaciones que se hacen alrededor de la violencia y que mantengan en silencio esa realidad como parte de su educación y condición de sumisión generacional.

El efecto de la baja escolaridad sobre la incidencia de actos violentos hacia las mujeres no puede desvincularse de la pobreza y la marginación. ${ }^{20}$ No obstante, la violencia también se manifiesta en las poblaciones educadas. El hallazgo de violencia hacia las mujeres con mayor instrucción sin duda refleja conflictos de género vinculados a la competencia de las mujeres por los espacios laborales, mejores niveles de ingreso y grados de autonomía asociados a una mayor escolaridad. Esto se hizo más manifiesto en las mujeres derechohabientes. Otra posibilidad también es que, al contar con mejores niveles de educación, respondan a la violencia con la denuncia más que las mujeres menos educadas. El impacto de la violencia también es visible en el estado civil de las parejas, pues las mujeres casadas y en unión libre la padecen con mayor reiteración y, en una proporción no conocida pero seguramente considerable, en las mujeres separadas y divorciadas puede haber sido determinante para romper el vínculo conyugal en vista de su frecuencia.

La frecuencia en el consumo de alcohol en los hombres puede contemplarse como un indicador de manejo del estrés condicionado por las situaciones laborales, de ingreso, oportunidades de desarrollo, satisfacción familiar, etc. ${ }^{21}$ Adicionalmente, el consumo de alcohol por la pareja emerge como uno de los riesgos más importantes y predictor de la incidencia de la violencia en la mujer. La frecuencia en el consumo está directamente asociada a la severidad y confluencia de varios tipos de violencia y confirma los resultados en otros estudios. ${ }^{11-13,15,22}$

\section{Conclusiones}

Estos resultados demuestran que la violencia forma parte de la vida en sociedad y que sus manifestaciones en todos lados son parecidas, aunque su severidad esté moldeada por las circunstancias particulares del grupo estudiado. En el caso de México indican que la violencia contra las mujeres permea todos los estratos sociales, representa algunas características de la cultura nacional y subraya la asignación del género como la construcción subjetiva de las identidades femenina y masculina, las relaciones de poder y las desigualdades entre hombres y mujeres. ${ }^{23}$ Abordar el tema de la violencia como un problema de salud pública refrenda el interés de las instituciones de salud de velar por el bienestar de las familias dentro y fuera del entorno 
doméstico. Como ningún otro problema de salud, la violencia obliga a ir desvelando una realidad que dejó de estar encerrada en el terreno de lo privado para volverse de interés público. A sabiendas de que las determinantes de la violencia en el escenario social se confunden con los patrones culturales y del comportamiento y se mezclan con las condiciones económicas, al ser identificadas deben permitir implantar estrategias más eficaces para atender y prevenir este problema de magnitud mundial. La Encuesta Nacional de la Violencia contra las Mujeres elaborada por la Secretaría de Salud constituye un esfuerzo por enfrentar esta realidad, al mostrar los datos que eliminan cualquier duda sobre la magnitud del problema en la sociedad mexicana. El deber de las instituciones es enfrentarlo para prevenir mayores daños a la salud de las mujeres mexicanas.

Dentro de las propuestas que se desprenden del análisis de la ENVIM, es posible enumerar algunas que ameritan ponerse en práctica de manera inmediata. En primer lugar, es indispensable realizar una capacitación masiva del personal de salud en todas las instituciones para motivar la identificación del problema en las mujeres usuarias de los servicios de salud. Este problema es de notificación obligatoria y, por lo mismo, requiere de la sensibilización del personal para enfrentarlo con empatía y responsabilidad. En segundo lugar, es importante su detección para conocer la magnitud real del problema y fortalecer las redes de apoyo a las mujeres maltratadas en los planos asistencial, legal, educativo y médico. Si bien el IMSS cuenta ya con iniciativas de detección de las mujeres que sufren de violencia, es importante intensificar las acciones para mejorar la cobertura y la comunicación con las mujeres afectadas. Además, se necesita intensificar la comunicación y los contactos con aquellas instituciones y organismos no gubernamentales dedicados a la atención de la violencia para mejorar la atención integral de las mujeres afectadas. Las tenues diferencias de la violencia en las mujeres derechohabientes y el resto de las mujeres encuestadas describen un problema que permea a todos los grupos sociales, es igualmente grave en todos los sectores y trastorna la dinámica familiar de toda la sociedad. De ahí que se deba enfrentar como un problema de interés nacional y que todo el sector salud lo integre a su agenda institucional como un programa prioritario. De los datos obtenidos se espera contribuir al establecimiento de algunas acciones que permitan identificar el impacto negativo que tiene la violencia en la salud de las mujeres derechohabientes del IMSS y con ello promover acciones para su atención.

\section{Referencias}

I. Sorenson S.Violence and injury in marital arguments: Risk patterns and gender differences. Am J Public Health, 1996;86:35-40.

2. Díaz-Martínez A, Esteban-Jiménez R.Violencia intrafamiliar Gac Med Mex 2003;139(4):353-355.

3. Díaz-Michel E, de la Garza AE.Violencia intrafamiliar: frecuencia y características en ambientes urbano y rural. Gac Med Mex 2003; 139(4):356-36I.

4. Sauceda GJM, Castillejos VG, Maldonado DJM,Violencia doméstica. El maltrato a la mujer. Gac Med Mex 2003;139(4):362-367.

5. Ramírez-Rodríguez JC, Uribe-Vázquez G. Mujer y violencia: un hecho cotidiano. Salud Publica Mex 1993;35:148-160.

6. Widding HL. Physical and sexual abuse against women and children.

Curr Opin Obstet Gynecol 2000;12:349-355.

7. Gazmararian JA, Lazorick S, Spitz AM, Ballard TJ, Saltzman LE, Marks SJ. Prevalence of violence against pregnant women.JAMA 1996;275:1915-1920. 8. Hijar-Medina M, Flores R.L,Valdez SR, Blanco J.Atención médica de lesiones intencionales provocadas por la violencia familiar. Salud Publica Mex 2003;45:252-258.

9. Organización Mundial de la Salud. Organización Mundial de la Salud. Informe sobre la salud en el mundo 2002. Reducir los riesgos y promover una vida sana. Ginebra: Ginebra: OMS, 2002: 84-86.

10. Freund KM, Bak SM, Blackhall L. Identifying domestic violence in primary care practice.J Gen Intern Med 1996; I :44-6.

II. Dearwater SR, Cohen JH, Campbell JC et al. Prevalence of intimate partner abuse in women treated at community hospital emergency departments. JAMA 1998;280:433-8.

12.Alpert EJ, Cohen S, Sege RD. Family violence: an overview. Acad Med 1997;72(I Suppl):S3-S6.

13. Hijar-Medina M, López-López NW, Blanco Muñoz J. La violencia y sus repercusiones en la salud. Reflexiones teóricas y magnitud del problema en México. Salud Publica Mex 1997;39:565-572.

I4. Eisenstat SA, Bancroft L. Domestic violence, N Engl J Med 1999;341:886-92.

15.Valdez R, Sanín LH. La violencia doméstica durante el embarazo y su relación con el bajo peso al nacer. Salud Publica Mex 1996;38:352-362. 16. Valdez R, Juárez C. Impacto de la violencia doméstica en la salud mental de las mujeres: análisis y perspectivas en México. Salud Mental 1998;21:1-10.

17. Olaiz G, Rico B, Del Río A. Encuesta Nacional sobre Violencia contra las Mujeres (ENVIM, 2003). Metodología de la encuesta. Cuernavaca, México: INSP/ SSA, 2003: 21-31.

18. Alvarado-Zaldívar G, Salvador-Moysén J, Estrada-Martínez S, Terrones-González A et al. Prevalencia de violencia doméstica en la ciudad de Durango. Salud Publica Mex 1998;40:48I-486.

19. Instituto Nacional de Estadística Geografía e Informática. Encuesta sobre violencia intrafamiliar, documento metodológico y resultados. México: INEGI, 2000.

20. Rivera-Rivera L, Lazcano-Ponce E, Salmerón-Castro J et al. Prevalence and determinants of male partner violence against Mexican women: A population-based study. Salud Publica Mex 2004;46(2): II3-122.

21. Horwitz A, Raskin WH, Howell-White S. The use of multiple outcomes in stress research: a case study of gender differences in responses to marital dissolution.J Health Soc Behaviour 1996;37:278-29I.

22. Medina-Mora ME, Berenzon S, Natera G. El papel del alcoholismo en las violencias. Gac Med Mex 1999;135:282-287.

23. Keijzer B. El género y el proceso salud-enfermedad-atención. Género y Salud en Cifras, 2003;I(3): 3-7. 